\title{
A CONVEX METRIC FOR A LOCALLY CONNECTED CONTINUUM
}

\author{
R. H. BING
}

A topological space is metrizable if there is a distance function $D(x, y)$ such that if $x, y, z$ are points, then

$$
\begin{gathered}
D(x, y) \geqq 0, \text { the equality holding only if } x=y, \\
D(x, y)=D(y, x) \text { (symmetry), } \\
D(x, y)+D(y, z) \geqq D(x, z) \text { (triangle condition), } \\
D(x, y) \text { preserves limit points. }
\end{gathered}
$$

By (4) we mean that $x$ is a limit point of the set $T$ if and only if for each positive number $\epsilon$ there is a point of $T$ at a positive distance from $x$ of less than $\epsilon$. We say that the metric $D(x, y)$ is convex if for each pair of points $x, y$ there is a point $u$ such that

$$
D(x, u)=D(u, y)=D(x, y) / 2 .
$$

A subset $M$ of a topological space $S$ is said to have a convex metric (even though $S$ may have no metric) if the subspace $M$ of $S$ has a convex metric.

It is known [5 [ ${ }^{1}$ that a compact continuum is locally connected if it has a convex metric. The question has been raised [5] as to whether or not a compact locally connected continuum $M$ can be assigned a convex metric. Menger showed [5] that $M$ is convexifiable if it possesses a metric $D$ such that for each point $p$ of $M$ and each positive number $\epsilon$ there is an open subset $R$ of $M$ containing $p$ such that each point of $R$ can be joined in $M$ to $p$ by a rectifiable arc of length (under $D$ ) less than $\epsilon$. Kuratowski and Whyburn proved [4] that $M$ has a convex metric if each of its cyclic elements does. Beer considered [1] the case where $M$ is one-dimensional. Harrold found [3] $M$ to be convexifiable if it has the additional property of being a plane continuum with only a finite number of complementary domains.

We shall show that if $M_{1}$ and $M_{2}$ are two intersecting compact continua with convex metrics $D_{1}$ and $D_{2}$ respectively, then there is a convex metric $D_{3}$ on $M_{1}+M_{2}$ that preserves $D_{1}$ on $M_{1}$ (Theorem 1). Using this result, we show that any compact $n$-dimensional locally connected continuum has a convex metric (Theorem 6). We do not 1948.

Presented to the Society, February 28, 1948; received by the editors June 21,

${ }^{1}$ Numbers in brackets refer to the references cited at the end of the paper. 
answer the question: Does each compact locally connected continuum have a convex metric? ${ }^{2}$

In my paper Extending a metric it is shown [2, Theorem 5] that if $K$ is a closed subset of the metrizable space $S$ and $D_{1}$ is a metric on $K$, then there is a metric $D_{2}$ on $S$ that preserves $D_{1}$ on $K$. The following result is a modification of that result.

THEOREM 1. If $M_{1}$ and $M_{2}$ are two intersecting compact continua with convex metrics $D_{1}$ and $D_{2}$ respectively, there is a convex metric $D_{3}$ on $M_{1}+M_{2}$ that preserves $D_{1}$ on $M_{1}$.

Proof. Let $G(x)$ be the least upper bound of $D_{1}(p, q)$ for all points $p, q$ of $M_{1} \cdot M_{2}$ such that $D_{2}(p, q) \leqq x$. Then

$$
G\left[D_{2}(p, q)\right] \geqq D_{1}(p, q) \quad\left(p, q \text { elements of } M_{1} \cdot M_{2}\right) .
$$

Since $G(x)$ is a monotone nondecreasing function of $x$ that approaches zero as $x$ approaches zero, there is a function $F(x)(x>0)$ such that $F(x)$ approaches zero as $x$ approaches zero, $F(x) \geqq G(x)$, and the derivative of $F(x)$ with respect to $x$ is a continuous monotone nonincreasing function greater than one.

If $C$ is an arc which lies except for possibly its end points in $M_{2}-M_{1} \cdot M_{2}$ and $\int_{C} F^{\prime}\left[D_{2}\left(p, M_{1}\right)\right] d s$ exists, where $F^{\prime}(x)$ represents the derivative of $F(x)$ with respect to $x, p$ is a variable point of $C, s$ is the length along $C$ under $D_{2}$, and $D_{2}\left(p, M_{1}\right)$ is the greatest lower bound of $D_{2}(p, q)$ for all points $q$ of $M_{1}$, we define the length $L_{0}(C)$ of $C$ under $D_{0}$ to be $L_{0}(C)=\int_{C} F^{\prime}\left[D_{2}\left(p, M_{1}\right)\right] d s$. We note that $L_{0}(C)$ is not defined for all arcs $C$. However, if $r$ and $q$ are two points which lie on an arc $C$ with such a length $L_{0}(C)$, then we call $D_{0}(r, q)$ the greatest lower bound of $L_{0}(C)$ for all such $\operatorname{arcs} C$ from $r$ to $q$. Since $F^{\prime}(x)>1, D_{0}(r, q)$ $\geqq D_{2}(p, q)$.

If $r$ and $q$ are two points of $M_{1}$ and $D_{0}(r, q)$ is defined, then

$$
D_{0}(r, q) \geqq D_{1}(r, q)
$$

because for each positive number $\epsilon$ there is a curve $C$ from $r$ to $q$ whose interior lies in $M_{1}-M_{1} \cdot M_{2}$ such that

$$
\begin{aligned}
D_{0}(r, q)+\epsilon & >\int_{C} F^{\prime}\left[D_{2}\left(p, M_{1}\right)\right] d s \geqq \int_{C} F^{\prime}(s) d s \\
& =F\left(\text { length } C \text { under } D_{2}\right) \geqq G\left[D_{2}(r, q)\right] \geqq D_{1}(r, q) .
\end{aligned}
$$

The first " $\geqq$ " relationship in (8) follows from the facts that $F^{\prime}(x)$ is a

${ }^{2}$ Since this paper was submitted, both E. E. Moise [8] and the author [9], working independently, have answered this question in the affirmative. 
nonincreasing function and $s \geqq D_{2}\left(p, M_{1}\right)$ since the end points of $C$ lie on $M_{1}$; the second " $\geqq$ " results from the facts that $F(x) \geqq G(x)$, length $C$ under $D_{2} \geqq D_{2}(r, q)$, and $G(x)$ is a monotone nondecreasing function; the third " $\geqq$ " is a consequence of (6).

If $q$ is a point of $M_{2}-M_{1} \cdot M_{2}$ and $C$ is a shortest arc from $q$ to $M_{1}$ under $D_{2}$, then

$$
L_{0}(C)=\int_{C} F^{\prime}\left[D_{2}\left(p, M_{1}\right)\right] d s=\int_{C} F^{\prime}(s) d s=F\left[D_{2}\left(q, M_{1}\right)\right] .
$$

Hence, if $p_{1}, p_{2}, \cdots$ is a sequence of points of $M_{2}-M_{1} \cdot M_{2}$ such that $D_{2}\left(p_{i}, M_{1}\right)$ approaches zero as $i$ increases without limit, then $D_{0}\left(p_{i}, M_{1}\right)$ approaches zero as $i$ increases without limit.

If $p$ and $q$ are two points of $M_{1}$, we define $D_{3}(p, q)$ to be $D_{1}(p, q)$; if $p$ is a point of $M_{1}$ and $q$ is a point of $M_{2}-M_{1} \cdot M_{2}$, we define $D_{3}(p, q)$ to be the greatest lower bound of $D_{1}(p, a)+D_{0}(a, q)$ for all points $a$ of $M_{1}$; if both $p$ and $q$ are points of $M_{2}-M_{1} \cdot M_{2}$, we define $D_{3}(p, q)$ to be the minimum of $D_{0}(p, q)$ and the greatest lower bound of $D_{0}(p, a)+D_{1}(a, b)+D_{0}(b, q)$, where $a$ is a point of $M_{1}$ and so is $b$. If $p=q$, we define $D_{3}(p, q)$ to be equal to zero.

The above definition of $D_{3}(p, q)$ is equivalent to defining $D_{3}(p, q)$ to be the greatest lower bound of the lengths of all arcs $C$ from $p$ to $q$ where length in $M_{1}$ is measured under $D_{1}$ and length in $M_{2}-M_{1} \cdot M_{2}$ is measured under $D_{0}$. It follows from (7) that we need only consider those arcs $C$ which intersect $M_{1}$ in a connected piece if at all.

Now the function $D_{3}(p, q)$ may be shown to satisfy conditions (1), (2), (3), (4), and (5). Hence, it is a convex metric for $M_{1}+M_{2}$ that preserves $D_{1}$ on $M_{1}$.

THEOREM 2. If $D_{1}$ and $D_{2}$ are convex metrics for the intersecting compact continua $M_{1}$ and $M_{2}$ respectively and $D_{2} \geqq D_{1}$ on $M_{1} \cdot M_{2}$, then for each positive number $\epsilon$ there is a convex metric $D_{3}$ for $M_{1}+M_{2}$ such that $D_{3}=D_{1}$ on $M_{1}, D_{3} \leqq D_{2}$ on $M_{2}$, and the diameter under $D_{3}$ of each component of $M_{2}-M_{1} \cdot M_{2}$ is less than $\epsilon$ plus twice the diameter under $D_{1}$ of the boundary with respect to $M_{1}+M_{2}$ of this component.

Proof. Define $E(p, q)$ to be the greatest lower bound of all sums of the type $f\left(p, p_{1}\right)+f\left(p_{1}, p_{2}\right)+\cdots+f\left(p_{n}, q\right)$ where adjacent points of $p, p_{1}, \cdots, p_{n}, q$ belong to the same one of the continua $M_{1}, M_{2}$ and $f\left(p_{i}, p_{j}\right)$ is $D_{1}\left(p_{i}, p_{j}\right)$ or $D_{2}\left(p_{i}, p_{j}\right)$ according as $p_{i}+p_{j}$ is or is not a subset of $M_{1}$. Since $D_{2} \geqq D_{1}$ on $M_{1} \cdot M_{2}, n$ need be no larger than 2 for $E(p, q)$ to attain this greatest lower bound. The convex metric $E$ on $M_{1}+M_{2}$ preserves $D_{1}$ on $M_{1}$ and $E \leqq D_{2}$ on $M_{2}$.

Let $X$ be the set of all points $p$ of $M_{2}-M_{1} \cdot M_{2}$ such that the 
distance from $p$ to $M_{1}$ under $E$ is greater than one-half the diameter under $E$ of the boundary with respect to $M_{1}+M_{2}$ of the component of $M_{2}-M_{1} \cdot M_{2}$ containing $p$.

Let $n$ be a positive number so small that $\epsilon / n$ is greater than twice the diameter of $M_{2}$ under $E$. If $C$ is a rectifiable (under $E$ ) arc in $M_{1}+M_{2}$ from $p$ to $q$, define $L(C)$ to be the greatest lower bound of all sums of the type $f\left(p, p_{1}\right)+f\left(p_{2}, p_{3}\right)+\cdots+f\left(p_{k}, q\right)$ where $p_{1}, p_{2}, \cdots$, $p_{k}$ are points of $C$ and $f\left(p_{i}, p_{j}\right)$ is either $n$ times or 1 times the length under $E$ of the subarc of $C$ from $p_{i}$ to $p_{j}$ according as this subarc is or is not a subset of $X$. If $D_{3}(p, q)$ is defined to be the greatest lower bound of all such values $L(C), D_{3}$ satisfies the conditions of Theorem 2.

THEOREM 3. Suppose $M_{2}$ is a compact continuum with a convex metric, $M_{2}$ lies in a complete locally connected space $S$ with a metric $D$, each component of $S-M_{2}$ is of diameter under $D$ of less than $\theta$ and $M_{1}$ is a subcontinuum of $M_{2}$ with a convex metric $D_{1}$ such that

$$
D_{1}(p, q) \leqq D(p, q) \quad \text { if } D_{1}(p, q)>\theta\left(p, q \text { elements of } M_{1}\right) \text {. }
$$

For each positive number $\epsilon$ there is a continuum $M_{3}$ containing $M_{2}$ and a convex metric $D_{3}$ for $M_{3}$ such that $D_{3}$ preserves $D_{1}$ on $M_{1}$ and the boundary of each component of $S-M_{3}$ is of diameter less than $\theta+\epsilon$ under $D_{3}$.

Proof. By Theorem 1, there is a convex metric $D_{2}$ for $M_{2}$ that preserves $D_{1}$ on $M_{1}$. Let $n$ be an integer so large that

$$
n D_{2}(p, q)>D(p, q) \quad \text { if } D(p, q)>\epsilon / 8\left(p, q \text { elements of } M_{2}\right) \text {. }
$$

Let $X$ denote the collection of all pairs of points $(x, y)$ such that both $x$ and $y$ are points of the boundary of the same component of $S-M_{2}$ and

$$
n D_{2}(x, y)>\theta+\epsilon .
$$

There is a finite subcollection $X^{\prime}$ of $X$ such that for each element $(x, y)$ of $X$ there is an element $\left(x^{\prime}, y^{\prime}\right)$ of $X^{\prime}$ such that both $x^{\prime}$ and $y^{\prime}$ are accessible from the same component of $S-M_{2}$ and

$$
n D_{2}\left(x, x^{\prime}\right)+n D_{2}\left(y^{\prime}, y\right)<\epsilon / 2 \text {. }
$$

Let $C_{1}, C_{2}, \cdots, C_{j}$ be a finite collection of components of $S-M_{2}$ irreducible with respect to the property that for each element of $X^{\prime}$ there is an integer $i$ less than or equal to $j$ such that both points of this element of $X^{\prime}$ are accessible from $C_{i}$.

There is a dendron $T_{i}(i=1,2, \cdots, j)$ such that $T_{i}$ lies except for 
its ends in $C_{i}$ and the sum of the ends of $T_{i}$ is a finite subset $Y_{i}$ of the boundary of $C_{i}$ such that $Y_{i}$ contains all points of the sum of the elements of $X^{\prime}$ that are accessible from $C_{i}$ and for each point $q$ of the boundary of $C_{i}$ there is a point $r$ of $Y_{i}$ such that $n D_{2}(r, q)<\epsilon / 2$. Let $D\left(T_{i}\right)$ be a convex metric for $T_{i}$ such that if $p$ and $q$ are two end points of $T_{i}$, then

$$
\theta+\epsilon / 4<D\left(T_{i} ; p, q\right)<\theta+\epsilon / 2 .
$$

Let $E$ be a metric for $M_{2}+\sum T_{i}=M_{3}$ such that the distance between two points of $M_{3}$ under $E$ is the greatest lower bound of the lengths of arcs containing them where length is measured by $n D_{2}$ in $M_{2}$ and by $D\left(T_{i}\right)$ in $T_{i}$. If $(x, y)$ is an element of $X$, it follows from (12) and (13) that

$$
E(x, y)<\theta+\epsilon .
$$

The diameter of the boundary of each component of $S-M_{3}$ is less than $\theta+\epsilon$ under $E$, for suppose $p$ and $q$ are two points of this boundary; if $p+q$ is a subset of $M_{2}, E(p, q)<\theta+\epsilon$ by (11) and (14); if neither $p$ nor $q$ is a point of $M_{2}$, both belong to some $T_{i}$ whose diameter is less than $\theta+\epsilon / 2$; if $p$ is an interior point of a $T_{i}$ which does not contain $q$, there is an end point $r$ of $T_{i}$ such that $n D_{2}(r, q)<\epsilon / 2$ and then $E(p, q) \leqq D\left(T_{i} ; p, r\right)+n D_{2}(r, q)<\theta+\epsilon$.

We shall show that if $r$ and $s$ are two points of $M_{1}$, then $D_{1}(r, s)$ $\leqq E(r, s)$. Suppose this is not the case and that $r s$ is an arc from $r$ to $s$ in $M_{3}$ whose length is less than $D_{1}(r, s)$ under $E$. If $D_{1}(r, s) \leqq \theta$, then $r s$ is a subset of $M_{2}$ alone and

$$
E(r, s)=n D_{2}(r, s) \geqq D_{2}(r, s)=D_{1}(r, s) .
$$

Suppose $r s$ is not a subset of $M_{2}$ and $p_{1} p_{2}, p_{3} p_{4}, \cdots, p_{2 j-1} p_{2 j}$ are the subarcs of $r s$ which lie except for their end points in $M_{3}-M_{2}$ where $p_{2 i-1} p_{2 i}$ precedes $p_{2 i+1} p_{2 i+2}$ on $r s$ in the order from $r$ to $s$. Let $Z(t)$ be 0 or $t$ according as $t$ is less than $\epsilon / 8$ or not. If $D_{1}(r, s)>\theta$, it follows from (9), (3), and (10) that

$$
\begin{aligned}
& D_{1}(r, s) \leqq D(r, s) \leqq D\left(r, p_{1}\right)+D\left(p_{1}, p_{2}\right)+\cdots+D\left(p_{2 j}, s\right) \\
&< D\left(r, p_{1}\right)+\theta+D\left(p_{2}, p_{3}\right)+\cdots+\theta+D\left(p_{2 j}, s\right) \\
&< Z\left[D\left(r, p_{1}\right)\right]+Z\left[D\left(p_{2}, p_{3}\right)\right]+\cdots+Z\left[D\left(p_{2 j}, s\right)\right] \\
&+j \theta+(j+1) \epsilon / 8 \\
& \leqq n D_{2}\left(r, p_{1}\right)+n D_{2}\left(p_{2}, p_{3}\right)+\cdots+n D_{2}\left(p_{2 j}, s\right) \\
&+j(\theta+\epsilon / 4) \\
& \leqq \\
& \text { length } r s \text { under } E .
\end{aligned}
$$


It follows from (15) and (16) that $E(r, s) \geqq D_{1}(r, s)$.

It follows from Theorem 2 that there is a convex metric $D_{3}$ for $M_{3}$ such that $D_{3}=D_{1}$ on $M_{1}$ and $D_{3} \leqq E$. The boundary of each component of $S-M_{3}$ is of diameter less than $\theta+\epsilon$ under $D_{3}$ because it is under $E$.

Theorem 4. Suppose $M$ is a compact locally connected continuum such that if $p$ is a point contained in an open subset $R_{1}$ of $M$, there is an open subset $R_{2}$ of $R_{1}$ containing $p$ such that the boundary of $R_{2}$ with respect to $M$ is a subset of a subcontinuum of $M$ with a convex metric. Then $M$ has a convex metric.

Proof. Let $F$ be a metric for $M$. We shall show that there is a collection of continua $M_{1}, M_{2}, \cdots$ in $M$ and a collection of metrics $D_{1}, D_{2}, \cdots$ such that:

(a) $M_{i+1}$ contains $M_{i}$.

(b) $D_{i}$ is a convex metric for $M_{i}$.

(c) $D_{i+1}$ preserves $D_{i}$ on $M_{i}$.

(d) Under $F$, each component of $M-M_{i}$ is of diameter less than $1 / 4^{i}$.

(e) Under $D_{i}$, the boundary (with respect to $M$ ) of each component of $M-M_{i}$ is of diameter less than $1 / 4^{i}$.

(f) Under $D_{i+1}$, the common part of the $M_{i+1}$ and each component of $M-M_{i}$ is of diameter less than $3 / 4^{i}$.

First, we show that if $\epsilon$ is a positive number, there is a subcontinuum $W$ of $M$ with a convex metric such that each component of $M-W$ is of diameter less than $\epsilon$ under $F$. By the Heine-Borel Theorem, we find that there is a finite collection $G$ of subcontinua of $M$ such that each element of $G$ has a convex metric, for each point $p$ of $M$ there is an element $g$ of $G$ such that $p$ belongs either to $g$ or to a component of $M-g$ of diameter under $F$ of less than $\epsilon$, and the sum $W$ of the elements of $G$ is a continuum. Each component of $M-W$ is of diameter less than $\epsilon$ under $F$ and it follows from Theorem 1 that $W$ has a convex metric.

Let $M_{1}$ be a subcontinuum of $M$ with a convex metric $E$ such that each component of $M-M_{1}$ is of diameter less than $1 / 4$ under $F$. A suitable multiple of $E$ gives a metric $D_{1}$ for $M_{1}$ which will satisfy conditions (b) and (e).

Let $n$ be an integer so large that

$$
n F(p, q)>D_{1}(p, q) \quad \text { if } \quad D_{1}(p, q)>1 / 17 \quad\left(p, q \text { elements of } M_{1}\right) .
$$

There is a continuum $W$ in $M$ containing $M_{1}$ such that $W$ has a convex metric and the diameter of each component of $M-W$ is less than 
$1 / 17$ under $n F$. By Theorem 3 , there is a continuum $M_{2}$ with a convex metric $E$ such that $M_{2}$ contains $W, E$ preserves $D_{1}$ on $M_{1}$, and the diameter under $E$ of the boundary with respect to $M$ of each component of $M-M_{1}$ is less than $1 / 4^{2}$. Applying Theorem 2 we find that $M_{2}$ has a convex metric $D_{2}$ such that $M_{2}$ and $D_{2}$ satisfy conditions (a), (b), (c), (d), (e), and (f).

Similarly, there exist continua $M_{3}, M_{4}, \cdots$ and convex metrics $D_{3}, D_{4}, \cdots$ satisfying conditions (a), (b), (c), (d), (e), and (f). Let $D$ be a function of the pairs of points of $M$ such that $D(p, q)$ is the lower limit of $D_{1}\left(p_{1}, q_{1}\right), D_{2}\left(p_{2}, q_{2}\right), \cdots$ where $p_{1}, p_{2}, \cdots$ and $q_{1}, q_{2}, \cdots$ are sequences of points converging to $p$ and $q$ respectively and $p_{i}+q_{i}$ is a subset of $M_{i}$. We shall show that $D$ preserves limit points in $M$.

Let $\epsilon$ be a positive number and $n$ an integer so large that $3 / 4^{n}$ $+3 / 4^{n+1}+\cdots<\epsilon / 4$. If $p$ and $q$ are points of the same component of $M-M_{n}$, then $D(p, q)<\epsilon / 2$ by (f). If $p$ is a point of $M_{n}$, let $R$ be the set of all points $r$ of $M_{n}$ such that $D_{n}(p, r)<\epsilon / 2$. The sum $V$ of $R$ and all components of $M-M_{n}$ that have a point of $R$ on their boundaries is an open subset of $M$ containing $p$ and if $q$ is a point of $V$, then $D(p, q)<\epsilon$. Hence, the set of points $q$ such that $D(p, q)>\epsilon$ is not a limit point of $p$.

If $V$ is an open subset of $M$ containing $p$, we shall show that there is a positive number $\epsilon$ such that $D(p, M-V) \geqq \epsilon$. Let $R$ be an open subset of $V$ containing $p$ and $n$ an integer such that $F(R, M-V)>3 / 4^{n}$. There is a positive number $\epsilon$ such that $D_{n}(r, s)>\epsilon$ if $F(r, s)>1 / 4^{n}$. Since each component of $M-M_{n}$ is of diameter less than $1 / 4^{n}$ under $F$, each arc in $M$ from $R$ to $M-V$ contains points $r$ and $s$ of $M_{n}$ such that $F(r, s)>1 / 4^{n}$. Hence, if $k$ is an integer bigger than $n$, $D_{k}\left(R \cdot M_{k},[M-V] \cdot M_{k}\right)>\epsilon$. Hence, $D(p, M-V) \geqq \epsilon$.

We have shown that $D$ satisfies conditions (1), (2), and (4). As each $D_{i}$ satisfies conditions (3) and (5) and $D$ is the limit of $D_{1}, D_{2}, \cdots$, then $D$ satisfies these conditions. Hence, it is a convex metric for $M$.

THEOREM 5. If $M$ is an n-dimensional locally connected compact continuum and $\epsilon$ is a positive number, there is a locally connected continuum $W$ in $M$ such that each component of $M-W$ is of diameter less than $\epsilon, W$ is $(n-1)$-dimensional if $n>1$, and $W$ is a dendron (acyclic continuous curve) if $n=1$.

Proof. An application of the Heine-Borel Theorem gives that there is an $(n-1)$-dimensional closed subset $H$ of $M$ such that each component of $M-H$ is of diameter less than $\epsilon$. If $n-1>0, H$ is 
contained in an ( $n-1)$-dimensional locally connected subcontinuum of $M[6$, Theorem 1]. If $n-1=0$, a dendron in $M$ contains $H$ [7, Theorem 1].

THEOREM 6. Each n-dimensional compact locally connected continuum has a convex metric.

Proof. If $n=1$, Theorem 6 follows from Theorems 4 and 5 and the fact that a dendron has a convex metric. If $n>1$, Theorem 6 follows from Theorems 4 and 5 and induction on $n$.

Definition. A set $S$ is said to be finite-dimensional if for each point $p$ of $S$ and each open subset $R$ of $S$ containing $p$ there is an integer $n$ and an open subset $R^{\prime}$ of $R$ containing $p$ such that the boundary of $R^{\prime}$ with respect to $S$ is $n$-dimensional.

The following result may be established by using Theorems 4,5 , and 6 .

THEOREM 7. Each finite-dimensional compact locally connected continuum has a convex metric.

\section{REFERENCES}

1. Gustav Beer, Beweis des Satzes, dass jede im kleinen zusammenhängende Kurve konvex metrisiert werden kann, Fund. Math. vol. 31 (1938) pp. 281-320.

2. R. H. Bing, Extending a metric, Duke Math. J. vol. 14 (1947) pp. 511-519.

3. O. G. Harrold, Jr., Concerning the convexification of continuous curves, Amer. J. Math. vol. 61 (1939) pp. 210-216.

4. C. Kuratowski and G. T. Whyburn, Sur les élêments cycliques et leurs applications, Fund. Math. vol. 16 (1930) pp. 305-331.

5. K. Menger, Untersuchungen über allgemeine Metrik, Math. Ann. vol. 100 (1928) pp. 75-163.

6. R. L. Wilder, On the imbedding of subsets of a metric space in Jordan continua, Fund. Math. vol. 19 (1932) pp. 45-64.

7. Leo Zippin, $A$ study of continuous curves and their relation to the JaniszerwskiMullikin Theorem, Trans. Amer. Math. Soc. vol. 31 (1929) pp. 744-770.

8. E. E. Moise, Grille decomposition and convexification theorems for compact locally connected continua, to appear in Bull. Amer. Math. Soc.

9. R. H. Bing, Partitioning a set, to appear in Bull. Amer. Math. Soc.

UNIVERSITY OF WISCONSIN 